\title{
Respuesta diferida a rituximab de una enfermedad hemolítica por crioaglutininas
}

\author{
J. M. CALVO-VILLAS, J. CUESTA TOVAR, E. CARRETER DE GRANDA, \\ F. SICILIA GUILLÉN \\ Servicio de Hematología y Hemoterapia. Hospital General de Lanzarote. Arrecife. \\ Lanzarote
}

\begin{abstract}
RESUMEN
El anticuerpo monoclonal anti-CD20 rituximab ha demostrado ser útil en el tratamiento de pacientes diagnosticados de enfermedad hemolítica severa por crioaglutininas. Presentamos nuestra experiencia con el empleo de rituximab en un paciente con hemólisis crónica por una enfermedad por crioaglutininas refractaria. Siete meses después de la administración del rituximab se detectó un aumento de la tasa de hemoglobina y durante el seguimiento durante 17 meses se mantuvo la respuesta hematológica. El rituximab puede jugar un papel en el tratamiento de individuos adultos con enfermedad por crioaglutininas refractarias a otros tratamientos con anemia dependientes de transfusión. Sin embargo, existen escasas series de casos que describan el empleo de rituximab en esta enfermedad hemolítica y son necesarios estudios prospectivos amplios que permitan aclarar el mejor esquema de administración, los posibles factores predictores del resultado, el beneficio de su asociación con otros fármacos y la posibilidad de obtener respuestas diferidas y mantenidas.
\end{abstract}

PALABRAS CLAVE: Enfermedad por crioaglutininas. Rituximab. Anemia hemolítica.
DELAYED RESPONSE TO RITUXIMAB OF COLD AGGLUTININ HAEMOLYTIC DISEASE

\begin{abstract}
The anti-CD20 monoclonal antibody rituximab has shown benefit in some patients diagnosed of severe cold agglutinin disease. Here we report our experience with rituximab in a patient with chronic haemolysis due to refractory cold agglutinin disease. An increase in the haemoglobin level was observed seven months later from rituximab administration and with a follow-up of 17 months, the patient maintains the haematological response. We suggest that rituximab can play an important role in the treatment of adult patients with refractory cold agglutinin disease with anaemia requiring transfusion. However only a few case reports of rituximab treatment in this haemolytic disease is available and there is need of large prospective studies that allow elucidate the better schedule of administration, the duration of the clinical effect, factors predictive of clinical outcome, the possible benefit of the association with other drugs and the possibility to achieve delayed and maintained responses.
\end{abstract}

KEY WORDS: Cold agglutinin disease. Rituxan. Hemolytic anemia.

Calvo-Villas JM, Cuesta Tovar J, Carreter de Granda E, Sicilia Guillén F. Respuesta diferida a rituximab de una enfermedad hemolítica por crioaglutininas. An Med Interna (Madrid) 2006; 23: 224-228.

\section{INTRODUCCIÓN}

La anemia hemolítica por anticuerpos fríos (AHAF) es una enfermedad de mecanismo autoinmune poco frecuente mediada por la presencia de autoanticuerpos del isotipo IgM que se fijan a antígenos eritrocitarias a bajas temperaturas (inferior a $37^{\circ} \mathrm{C}$ ) provocando la hemólisis por activación del complemento (1). Los tratamientos empleados para su control no han obtenido buenos resultados y la falta de respuesta o la reaparición de la anemia hemolítica es común en la mayoría de pacientes. El empleo del anticuerpo monoclonal anti-CD20 rituximab ha demostrado ser útil en diferentes citopenias autoinmunes refractarias a otras líneas de tratamiento y hay evidencias en la literatura sobre su efecto beneficioso en la enfermedad hemolítica por crioaglutininas.

El caso aportado es un paciente diagnosticado de anemia hemolítica por anticuerpos fríos refractaria a esteroides y con hiperglucemia sintomática secundaria al tratamiento esteroideo, que obtuvo criterios de respuesta hematológica a los siete meses de la administración del anticuerpo monoclonal antiCD20 y que mantiene sin tratamiento una cifra estable de hemoglobina superior a $10 \mathrm{~g} / \mathrm{dL}$, reducción del componente monoclonal IgM y cifras de bilirrubina indirecta normales con persistencia de valores de LDH altos a los 17 meses de seguimiento.

Trabajo aceptado: 29 de noviembre de 2005 


\section{CASO APORTADO}

Varón de 54 años de edad diagnosticado de anemia hemolítica por anticuerpos fríos en marzo de 1998 que consulta en febrero de 2004 por cianosis en partes acras, astenia severa, dolor lumbar y coluria de días de evolución

Entre sus antecedentes destacaba una osteoartropatía discal con mielopatía cervical y una diabetes mellitus tipo II no dependiente de insulina.

En marzo 1998 consulta por síndrome anémico y parestesias en manos y pies que aumentaban con el frío. A la exploración física palidez de piel y mucosas, sin adenopatías ni organomegalias. En las pruebas complementarias destacaba una hemoglobina $(\mathrm{Hb})$ de 11,9 $\mathrm{g} / \mathrm{dL}$ con autoaglutinación de los hematíes a temperatura ambiente que se corregían al procesar la muestra de sangre a una temperatura de $37{ }^{\circ} \mathrm{C}$. La fracción reticulocitaria era del 4\%; bioquímica con LDH de $483 \mathrm{U} / \mathrm{L}$ y la bilirrubina total de $2 \mathrm{mg} / \mathrm{dL}$ con la fracción indirecta (BI) de 1,9 $\mathrm{mg} / \mathrm{dL}$. La prueba de antiglobulina directa (PAD) fue positiva con suero poliespecífico $++++/++++$ y con monoespecífico para $\mathrm{C} 3 \mathrm{~d}$. El estudio de crioaglutininas detectó un anticuerpo anti-I a un título de 1/128. La prueba de antiglobulina indirecta a $4^{\circ}$ y a $37^{\circ}$ fue positiva. La haptoglobina sérica fue indetectable y la hemopexina de $45 \mathrm{mg} / \mathrm{dL}$. La dosificación de inmunoglobulinas, fracciones del complemento, detección de crioglobulinas, pruebas de autoinmunidad, factor reumatoide, niveles de vitamina B12 y ácido fólico (sérico e intraeritrocitario); serologías para sífilis, mycoplasma, parvovirus B19, virus de la hepatitis A, B y C, virus de la inmunodeficiencia humana, intradermoreacción de Mantoux y marcadores tumorales fueron normales o negativas. La radiografía de tórax, ecografía abdominal y una TAC de tórax y abdomen fueron normales. El paciente inició tratamiento con ácido fólico (5 mg/día) y se recomendaron medidas para evitar la exposición al frío.

Se mantuvo asintomático con cifras estables de hemoglobina, bilirrubina y LDH hasta julio de 2001 que consulta por cianosis en partes acras, anemia sintomática con $\mathrm{Hb}$ de $8 \mathrm{~g} / \mathrm{dL}$ y coluria, se transfunde un concentrado de hematíes y se administran 30 miligramos diarios de prednisona oral. La tolerancia es mala con episodios repetidos de hiperglucemia sintomática que requieren terapia con insulina y se cambia la prednisona por deflazacort oral $(12 \mathrm{mg} /$ día $)$. El recuento de inmunoglobulinas detecta una IgM de $286 \mathrm{mg} / \mathrm{dL}$ monoclonal. Un TAC corporal total no detecta adenopatías ni visceromegalias.
En junio de 2002 se objetivan datos clínicos de miopatía esteroidea y se disminuye la dosis de deflazacort oral a $6 \mathrm{mg} / \mathrm{d} i ́ a$. Se mantuvo la pauta de evitar la exposición al frío, ácido fólico oral y deflazacort hasta febrero de 2005. El paciente mantuvo crisis hemolíticas asociadas al descenso de temperatura ambiente con una $\mathrm{Hb}$ entre 6,5 $\mathrm{g} / \mathrm{dL}$ y $8,5 \mathrm{~g} / \mathrm{dL}$ con la prueba de la antiglobulina directa positiva requiriendo hemoterapia ocasional con concentrados de hematíes. Los valores de reticulocitos, $\mathrm{LDH}$, bilirrubina indirecta estaban altos y el componente monoclonal de IgM aumentaba de forma progresiva.

En febrero de 2004 consulta por acrocianosis dolorosa, anemia sintomática y coluria de días de evolución con una hemoglobina de 8 $\mathrm{g} / \mathrm{dL}, \mathrm{LDH}$ de $2.544 \mathrm{U} / \mathrm{L}$, bilirrubina indirecta de $2,21 \mathrm{mg} / \mathrm{dL}$ y una IgM monoclonal de $585 \mathrm{mg} / \mathrm{dL}$. Se inicia pauta descendente de corticoterapia hasta suspender y administración del protocolo terapéutico con rituximab intravenoso a dosis de $375 \mathrm{mg} / \mathrm{m}^{2}$ (x4 dosis) semanalmente según esquema de Ahrens (2). La evolución de los parámetros de hemólisis previos al tratamiento con rituximab se expone en la tabla I.

La respuesta al rituximab se obtuvo siete meses después de la administración del rituximab al objetivar un aumento significativo de la tasa de hemoglobina con reducción progresiva del componente IgM monoclonal (Fig. 1) permaneciendo los valores de LDH y del porcentaje de reticulocitos altos.

En agosto de 2005 el enfermo está asintomático con una hemoglobina estable superior a $10 \mathrm{~g} / \mathrm{dL}$ y sin crisis hemolíticas agudas relacionadas con el descenso de temperatura. La evolución de los parámetros de hemólisis después del tratamiento con rituximab se expone en la figura 2 .

\section{DISCUSIÓN}

La presencia de crioaglutininas con capacidad hemolítica se ha asociado con infecciones por mycoplasma, mononucleosis infecciosa o citomegalovirus, con síndromes linfoproliferativos y se han detectado autoanticuerpos fríos en enfermedades tumorales, autoinmunes o inmunodeficiencias $(1,3,4)$. La forma idiopática de la anemia hemolítica por crioaglutininas no está relacionada con ninguna enfermedad, sin embargo en la médula ósea de estos pacientes se ha detectado una prolife-

TABLA I

EVOLUCIÓN CLÍNICA DE LOS PARÁMETROS DE HEMÓLISIS Y DEL TRATAMIENTO PREVIO A LA ADMINISTRACIÓN DE RITUXIMAB

\begin{tabular}{|c|c|c|c|c|c|c|}
\hline & Diagnóstico & Crisis hemolítica & Intolerancia a prednisona & Intolerancia a esteroides & Crisis hemolitica & Crisis hemolítica \\
\hline Fecha & Marzo/98 & Julio/01 & Agosto/01 & Junio/02 & Mayo/03 & Marzo/04 \\
\hline Tratamiento* & No & $\begin{array}{l}\text { Hemoterapia } \\
\text { Prednisona }\end{array}$ & Deflazacort & Deflazacort & $\begin{array}{l}\text { Hemoterapia } \\
\text { Deflazacort }\end{array}$ & Rituximab \\
\hline Dosis (mg/dia) & - & 30 & 12 & 6 & 6 & $\begin{array}{c}375 \mathrm{mg} / \mathrm{m}^{2} \\
(\times 4 \text { dosis })\end{array}$ \\
\hline Hemoglobina ( $g / L)$ & 129 & 80 & 80 & 86 & 65 & 81 \\
\hline Bilirrubina indirecta $(\mathrm{mg} / \mathrm{dL})$ & 1,9 & 2 & 1,4 & 1,9 & 2 & 2,2 \\
\hline Haptoglobina (g/L) & Indetectable & Indetectable & Indetectable & Indetectable & Indetectable & Indetectable \\
\hline Paraproteína IgM (mg/dL) & 137 & 192 & 286 & 295 & 350 & 585 \\
\hline $\begin{array}{l}\text { Prueba de antiglobulina directa } \\
\text { Comentarios }\end{array}$ & t+t+ & t+++ & $\begin{array}{c}+++ \\
\text { Hiperglucemia } \\
\text { sintomática } \\
\text { Insulinoterapia }\end{array}$ & $\begin{array}{c}+++ \\
\text { Hiperglucemia } \\
\text { Miopatía } \\
\text { esteroidea }\end{array}$ & $\begin{array}{c}++++ \\
\text { Síndrome } \\
\text { anémico }\end{array}$ & $\begin{array}{c}++++ \\
\text { Síndrome } \\
\text { anémico }\end{array}$ \\
\hline
\end{tabular}

\footnotetext{
* Todos los pacientes evitaron la exposición al frío y recibieron de ácido fólico a dosis de 5 mg/día
} 


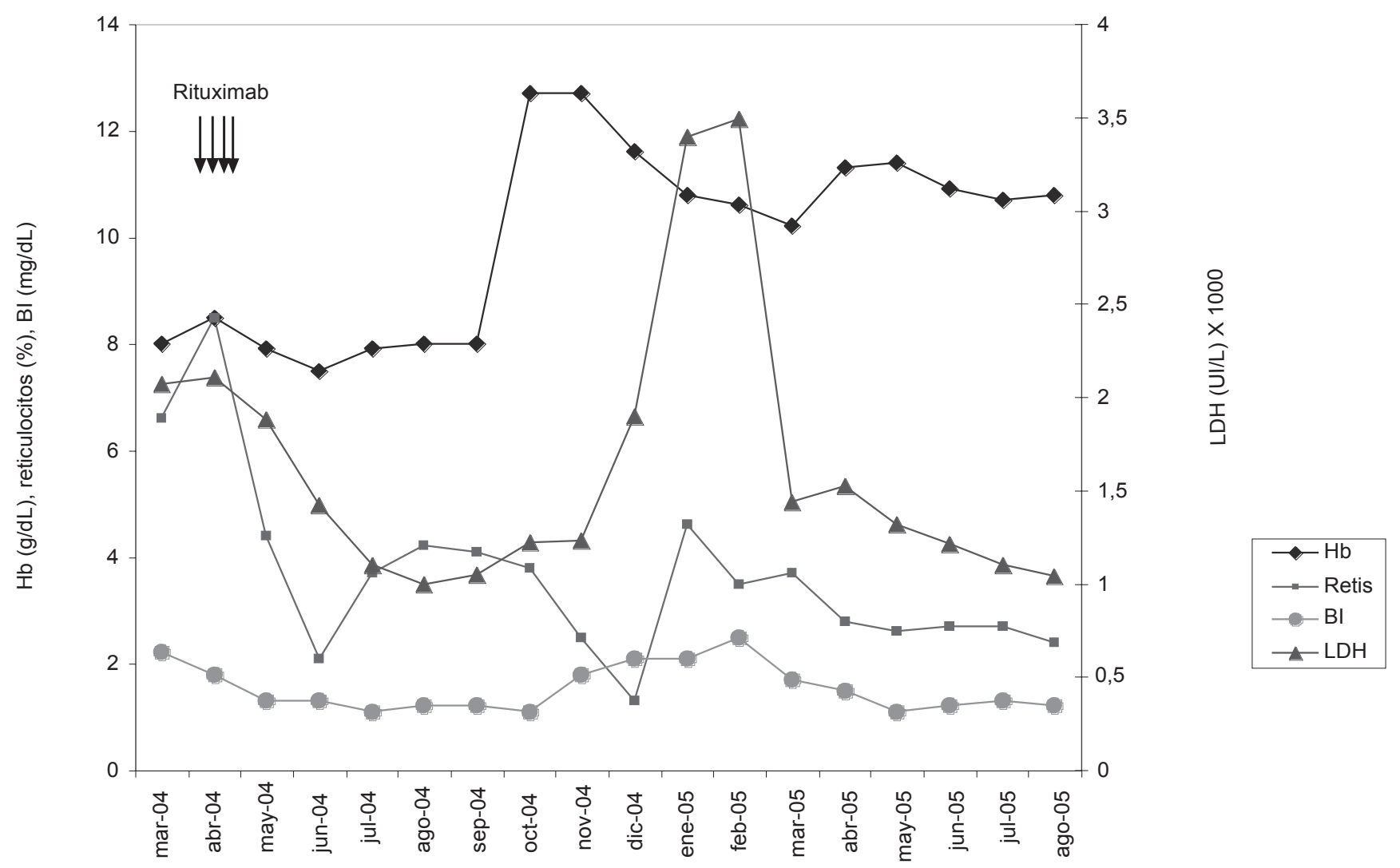

Fig. 1. Evolución de los parámetros de hemólisis después del tratamiento con rituximab.

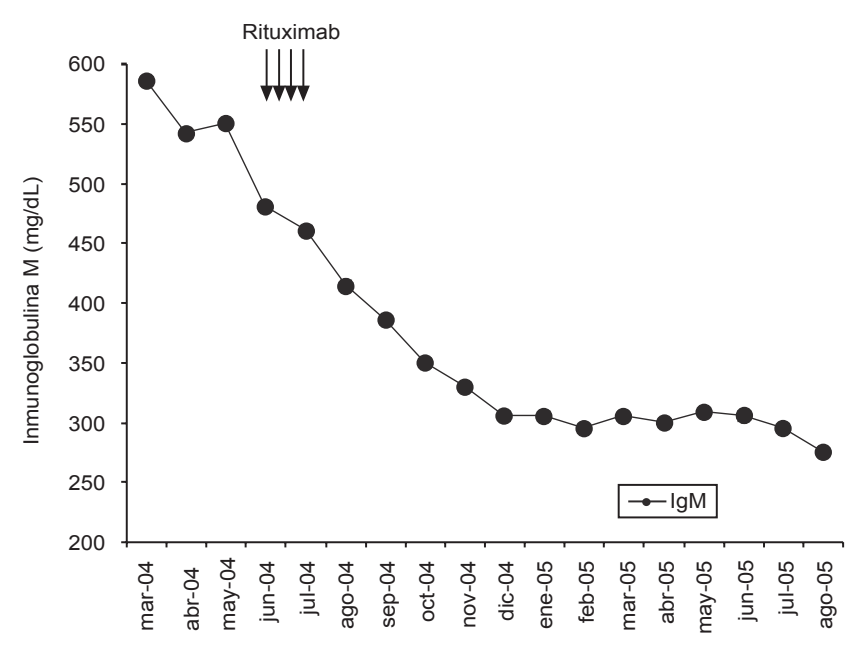

Fig. 2. Cambios en la cuantificación de IgM tras el tratamiento con rituximab.

ración clonal de linfocitos B similares a los del linfoma linfoplasmocitario que sintetizan una paraproteína monoclonal IgM tipo crioaglutinina con capacidad hemolítica $(3,5,6)$. Algunos casos de AHAF que progresan a una enfermedad linfoproliferativa B presentan en el cariotipo una trisomía del cromosoma 3 (7).
La respuesta de este tipo de anemia a los tratamientos clásicos como la prednisona, la quimioterapia citotóxica, la infusión de inmunoglobulinas intravenosas, la esplenectomía o sus diferentes asociaciones $(3,6,8,9)$ es insatisfactoria a lo que se une la morbilidad asociada a su uso crónico que puede llevar a un daño de la inmunidad celular y humoral y a un estado de inmunosupresión permanente (9). Las medidas más empleadas han sido mantener al paciente en ambiente templado asumiendo las incomodidades que produce en su calidad de vida, el uso de la plasmaféresis (10) en periodos cortos de tiempo y en algún caso se ha obtenido éxito con la fludarabina (11) y el anticuerpo monoclonal anti-CD52 alemtuzumab (12).

Los linfocitos B clonales juegan un papel clave en la patogénesis de la enfermedad hemolítica por anticuerpos fríos con un componente monoclonal sérico $(3,5,6)$ lo que supone que un procedimiento terapéutico que constituya un mecanismo de bloqueo específico de estos linfocitos B puede controlar la anemia hemolítica al suprimir la producción de autoanticuerpos con capacidad hemolítica.

El rituximab es un anticuerpo monoclonal anti-CD20 cuyas indicaciones están limitadas al tratamiento de síndromes linfoproliferativos como la leucemia linfática crónica y linfomas no hodgkianos con inmunofenotipo B y con expresión del antígeno de membrana CD20 (13). En los últimos años se ha ampliado su uso al tratamiento de diferentes citopenias autoinmunes (Tabla II) como la púrpura trombocitopénica autoinmune (14) y la anemia hemolítica autoinmune por diferentes isotipos de autoanticuerpos (15-22). Los mejores 
TABLA II

POSIBLES INDICACIONES PARA EL USO DE RITUXIMAB EN CITOPENIAS AUTOINMUNES (8)

\section{-Pacientes con enfermedad hemolítica por crioaglutini- nas con anemia dependiente de transfusión y/o sínto- mas relacionados con la enfermedad y también como terapia de primera línea (el tratamiento convencional suele ser ineficaz). \\ -Pacientes con púrpura trombocitopénica autoinmune con recuentos de plaquetas inferiores a $10 \times 109 / \mathrm{L}$ y/o sintomatología hemorrágica y refractarios a la terapia convencional. \\ -Pacientes con anemia hemolítica autoinmune por anti- cuerpos calientes, aplasia pura de células rojas o trom- bocitopenia y neutropenia inmune con criterios clínicos de gravedad y refractarios a la terapia convencional*}

* La evidencia en estas indicaciones es todavía limitada.

resultados del rituximab entre las diferentes citopenias autoinmunes se han conseguido en la enfermedad hemolítica por crioaglutininas (8). En la actualidad no existe un estudio comparativo aleatorizado de rituximab en la AHAF y las conclusiones se extraen de estudios con pocos pacientes prospectivos (6) o retrospectivos (23) que han encontrado eficacia del rituximab en la forma idiopática de la enfermedad hemolítica por crioaglutininas y también en la asociada a síndromes linfoproliferativos crónicos B (4,8,24-26).

El rituximab es un fármaco seguro y efectivo en la mayoría de las anemias hemolíticas autoinmunes con un índice de respuesta en la infancia superior al $80 \%(16,27)$. En adultos con enfermedad hemolítica por crioaglutininas refractaria las series con mayor número de pacientes han comunicado respuestas favorables en un porcentaje cercano al 50\% $(6,15,23)$.

La respuesta hematológica al rituximab en la anemia hemolítica refractaria por anticuerpos fríos ha permanecido estable con una $\mathrm{Hb}$ superior a $10 \mathrm{~g} / \mathrm{dL}$ sin necesitar transfusión en el 50\% de casos. Un 50\% de enfermos que recibieron rituximab tienen en el seguimiento un test de Coombs directo negativo (23).

La mediana para la obtención de respuesta al anticuerpo anti-CD20 está cercana a las 4 semanas, no existiendo ningún caso publicado con un tiempo de respuesta superior a las 14 semanas, la respuesta máxima se alcanza alrededor de los tres meses de la infusión del fármaco y la mediana de duración de la respuesta esta próxima al año $(6,21,23,28)$.

En este caso la aparición de la respuesta hematológica fue siete meses después de la administración del rituximab y once meses después del inicio de la respuesta mantiene una tasa de hemoglobina superior a $10 \mathrm{~g} / \mathrm{dL}$ sin transfusión con la prueba de antiglobulina directa positiva, ha presentado una crisis hemolítica leve sin repercusión en la cifra de $\mathrm{Hb}$ aunque los parámetros bioquímicos de hemólisis se mantienen elevados.

En nuestra experiencia, el anticuerpo monoclonal antiCD20 ha sido un tratamiento eficaz en el control de la anemia hemolítica por crioaglutininas no asociada a síndromes linfoproliferativos crónicos B refractaria e intolerante a la corticoterapia. La buena tolerancia a la administración y la falta de tratamientos alternativos efectivos convierten al rutiximab en una posibilidad terapéutica atractiva para las anemias hemolíticas por crioaglutininas refractarias. El seguimiento mayor de los pacientes con AHAF que hayan recibido rituximab permitirá establecer su papel real en esta enfermedad autoinmune.

La duración prolongada de las respuestas, que pueden llegar hasta tres años, y la posibilidad de utilizar el rituximab en las recaídas lo hacen un buen candidato para su evaluación en la AHAF.

El diseño de estudios prospectivos aleatorizados con rituximab en la anemia hemolítica por crioaglutininas establecerá la utilidad del anticuerpo anti-CD20 para las formas refractarias y en el tratamiento de primera línea (29), el número óptimo de ciclos para obtener la máxima respuesta, la utilidad del mantenimiento con rituximab en una enfermedad caracterizada por sus frecuentes recaídas (29) y la posibilidad de conseguir respuestas tardías que mantengan una hemoglobina superior a $10 \mathrm{mg} / \mathrm{dL}$ a largo plazo. Otros aspectos a determinar serían establecer la relación coste/efectividad, los factores predictores del resultado clínico y el posible beneficio de su asociación con otros tratamientos frente a la monoterapia como esquema inicial.

\section{Bibliografía}

1. Zilow G, Kirschfink M, Roelcke D. Red cell destruction in cold agglutinin disease. Infusionsther Transfusionsmed 1994; 21: 410-415.

2. Ahrens N, Kingreen D, Seltsam A, Salama A. Treatment of refractory autoimmune haemolytic anaemia with anti-CD20 (rituximab). Br J Haematol 2001; 114: 244-245.

3. Berentsen S, Bo K, Shammas FV, Myking AO, Ulvestad E. Chronic cold agglutinin disease of the "idiopathic" type is a premalignant or lowgrade malignant lymphoproliferative disease. APMIS 1997; 105: 354362.

4. Engelhardt M, Jakob A, Ruter B, Trepel M, Hirsch F, Lubbert M. Severe cold hemagglutinin disease (CHD) successfully treated with rituximab. Blood 2002; 100: 1922-1923.

5. Wendell F. Rosse, Peter Hillmen, and Alan D. Schreiber.ImmuneMediated Hemolytic Anemia. Hematology 2004: 48-62.

6. Berentsen S, Ulvestad E, Gjertsen BT, Hjorth-Hansen H, Langholm R, Knutsen H, et al. Rituximab for primary chronic cold agglutinin disease: a prospective study of 37 courses of therapy in 27 patients. Blood 2004;
103: 2925-2928.

7. Michaux L, Dierlamm J, Wlodarska I, Stul M, Bosly A, Delannoy A, et al. Trisomy 3 is a consistent chromosome change in malignant lymphoproliferative disorders preceded by cold agglutinin disease. Br J Haematol 1995; 91: 421-424.

8. Finazzi G. Rituximab in autoimmune cytopenias: for which patients? Haematologica 2002; 87: 113-114

9. Arzoo K, Sadeghi S, Liebman HA. Treatment of refractory antibody mediated autoimmune disorders with an anti-CD20 monoclonal antibody (rituximab). Ann Rheum Dis 2002; 61: 922-924.

10. Zoppi M, Oppliger R, Althaus U, Nydegger U. Reduction of plasma cold agglutinin titers by means of plasmapheresis to prepare a patient for coronary bypass surgery. Infusionsther Transfusionsmed 1993; 20: 1922.

11. Jacobs A. Cold agglutinin hemolysis responding to fludarabine therapy. Am J Hematol 1996; 53: 279-280.

12. Robak T. Monoclonal antibodies in the treatment of autoimmune cyto- 
penias. Eur J Haematol 2004; 72: 79-88.

13. Plosker GL, Figgitt DP. Rituximab: a review of its use in non-Hodgkin's lymphoma and chronic lymphocytic leukaemia. Drugs 2003; 63: 803843.

14. Kazkaz H, Isenberg D. Anti B cell therapy (rituximab) in the treatment of autoimmune diseases. Curr Opin Pharmacol 2004; 4: 398-402.

15. Shanafelt TD, Madueme HL, Wolf RC, Tefferi A. Rituximab for immune cytopenia in adults: idiopathic thrombocytopenic purpura, autoimmune hemolytic anemia, and Evans syndrome. Mayo Clin Proc 2003; 78: 1340-1346.

16. Quartier P, Brethon B, Philippet P, Landman-Parker J, Le Deist F, Fischer A. Treatment of childhood autoimmune haemolytic anaemia with rituximab. Lancet 2001; 358: 1511-1513.

17. Zecca M, De Stefano P, Nobili B, Locatelli F. Anti-CD20 monoclonal antibody for the treatment of severe, immune-mediated, pure red cell aplasia and hemolytic anemia. Blood 2001; 97: 3995-3997.

18. Ramanathan S, Koutts J, Hertzberg MS. Two cases of refractory warm autoimmune hemolytic anemia treated with rituximab. Am J Hematol 2005; 78: 123-126.

19. Wakim M, Shah A, Arndt PA, Garratty G, Weinberg K, Hofstra T, Church J. Successful anti-CD20 monoclonal antibody treatment of severe autoimmune hemolytic anemia due to warm reactive $\operatorname{IgM}$ autoantibody in a child with common variable immunodeficiency. Am J Hematol 2004; 76: 152-155.

20. Zaja F, Vianelli N, Sperotto A, Patriarca F, Tani M, Marin L, et al. AntiCD20 therapy for chronic lymphocytic leukemia-associated autoimmune diseases. Leuk Lymphoma 2003; 44: 1951-1955.

21. Zaja F, Iacona I, Masolini P, Russo D, Sperotto A, Prosdocimo S, et al. B-cell depletion with rituximab as treatment for immune hemoly- tic anemia and chronic thrombocytopenia. Haematologica 2002; 87: 189-195.

22. Barquero-Romero J, García Domínguez M, Pérez Miranda M. Respuesta mantenida al tratamiento con anticuerpos anti-CD20 (rituximab) en un caso de anemia hemolítica autoinmune asociado a artritis reumatoide. Med Clin (Barc) 2005; 124: 517-518.

23. Cabrera JR, Peñalver FJ, Millán I, Álvarez-Larran A, Diez-Martín JL, Gallur L, et al. Mabthera (Rituximab) in the Treatment of 34 Adult Patients with Refractory Autoimmune Hemolytic Anemia (AIHA). Blood 2004; 104: 1619 (abstract).

24. Zaja F, Russo D, Fuga G, Michelutti T, Sperotto A, Fanin R, et al. Rituximab in a case of cold agglutinin disease. Br J Haematol 2001; 115: 232-233.

25. Mori A, Tamaru J, Sumi H, Kondo H. Beneficial effects of rituximab on primary cold agglutinin disease refractory to conventional therapy. Eur J Haematol 2002; 68: 243-246.

26. Sparling TG, Andricevic M, Wass H. Remission of cold hemagglutinin disease induced by rituximab therapy. CMAJ 2001; 164: 1405.

27. Zecca M, Nobili B, Ramenghi U, Perrotta S, Amendola G, Rosito P, Jankovic M, Pierani P, De Stefano P, Bonora MR, Locatelli F. Rituximab for the treatment of refractory autoimmune hemolytic anemia in children. Blood 2003; 101: 3857-3861.

28. Gupta N, Kavuru S, Patel D, Janson D, Driscoll N, Ahmed S, Rai KR. Rituximab-based chemotherapy for steroid-refractory autoimmune hemolytic anemia of chronic lymphocytic leukemia. Leukemia 2002; 16: 2092-2095.

29. Pulik M, Genet P, Lionnet F, Touahri T. Treatment of primary chronic cold agglutinin disease with rituximab: maintenance therapy may improve the results. Br J Haematol 2002; 117: 998-999. 